\title{
Liver injury after antiviral treatment of critically ill patients with COVID-19: a single-centered retrospective cohort study
}

\author{
Xinjia Ruan ${ }^{1 \#}$, Xiaofan Lü ${ }^{1 \#}$ Kangyi Wang ${ }^{1}$, Bing Zhang ${ }^{2}$, Jun Wang ${ }^{3}$, Yongsheng Li $^{4}$, Jiashuo Wang ${ }^{1}$, \\ Zhengbao Xu', Fangrong Yan ${ }^{1}$ \\ ${ }^{1}$ State Key Laboratory of Natural Medicines, Research Center of Biostatistics and Computational Pharmacy, China Pharmaceutical University, \\ Nanjing, China; ${ }^{2}$ Department of Radiology, The Affiliated Nanjing Drum Tower Hospital of Nanjing University Medical School, Nanjing, China; \\ ${ }^{3}$ Department of Intensive Care Medicine, The First Affiliated Hospital of Soochow University, Suzhou, China; ${ }^{4}$ Department of Intensive Care \\ Medicine, Tongji Hospital, Tongji Medical College, Huazhong University of Science and Technology, Wuhan, China \\ Contributions: (I) Conception and design: X Ruan, X Lu; (II) Administrative support: Y Fan, B Zhang; (III) Provision of study materials or patients: \\ J Wang, Y Li; (IV) Collection and assembly of data: J Wang, Z Xu, K Wang; (V) Data analysis and interpretation: X Ruan; (VI) Manuscript writing: \\ All authors; (VII) Final approval of manuscript: All authors. \\ \#These authors contributed equally to this work. \\ Correspondence to: Fangrong Yan, PhD. State Key Laboratory of Natural Medicines, Research Center of Biostatistics and Computational Pharmacy, \\ China Pharmaceutical University, Nanjing 210009, China. Email: f.r.yan@163.com.
}

\begin{abstract}
Background: Severe acute respiratory syndrome coronavirus 2 (SARS-Cov-2) is the causative agent of coronavirus disease 2019 (COVID-19). Lung lesions are considered to be the main damage caused by SARS$\mathrm{CoV}-2$ infection. In addition, liver injury has also been reported to occur during the course of the disease in severe cases. However, the effect of antiviral treatment on liver injury in critically ill patients is not yet clear.

Methods: We retrospectively evaluated the effect of antiviral treatment and antiviral drug arbidol on liver injury in COVID-19 critically ill patients. Baseline characteristics were collected from patients who were admitted to intensive care units of Tongji Hospital in Wuhan, China, and confounders were balanced by propensity score matching (PSM) and inverse probability of treatment weighting (IPTW) analyses.

Results: Both the PSM (OR =2.77; 95\% CI: 1.03, 7.48; P=0.045) and the IPTW-adjusted (OR =2.33; 95\% CI: $1.02,5.34 ; \mathrm{P}=0.047)$ results showed that COVID-19 critically ill patients receiving antiviral treatment had a significantly higher risk of liver injury. However, arbidol treatment did not have a significant effect on liver injury (IPTW: OR =2.11; 95\% CI: 0.79, 5.67; P=0.14).

Conclusions: Our results show that although arbidol treatment does not seem to be significantly associated with liver injury complications, the overall use of antiviral drugs increases the risk of liver injury for critically ill patients with COVID-19. Antiviral drugs are widely used to treat COVID-19, but we recommend that for critically ill patients, antiviral treatment should be used with caution considering both effectiveness and potential adverse effects.
\end{abstract}

Keywords! Coronavirus disease 2019 (COVID-19); critically ill patients; antiviral treatment; liver injury

Submitted Aug 10, 2020. Accepted for publication Nov 12, 2020.

doi: 10.21037/apm-20-1581

View this article at: http://dx.doi.org/10.21037/apm-20-1581

\section{Introduction}

In December 2019, a new type of coronavirus, severe acute respiratory syndrome coronavirus 2 (SARS-CoV-2), broke out (1). At present, the spread of coronavirus disease 2019 (COVID-19) has reached the level of a global pandemic.
Most infected patients experience symptoms of pneumonia, such as fever, fatigue, difficulty breathing, and cough, while a large number of infected patients are asymptomatic. Although some patients are able to recover by themselves, this disease can also be fatal, with a mortality rate of 
about $3 \%$ (2). Seriously ill patients can die from massive alveolar damage and progressive respiratory failure (3). Approximately $5-10 \%$ of patients develop severe oxygen deficiency and require intensive care and mechanical ventilation through intubation $(4,5)$. Studies have shown that the incidence of liver injury was between $14.8 \%$ and $53 \%$ in patients with COVID-19 (6), which can be indicated by elevated levels of Lactate dehydrogenase $(\mathrm{LDH})$, aspartate aminotransferase (AST) and alanine aminotransferase (ALT) (7). Recent reports indicated that the incidence of liver injury was higher in severe COVID-19 cases than in patients with mild symptoms $(8,9)$. In death cases of COVID-19, the incidence of liver injury might reach as high as $78 \%$ (10).

The direct cause of liver injury in patients with coronavirus infection may be virus infection of liver cells. The virus may invade the liver through the digestive tract or blood circulation (11). SARS-CoV-2 enters target cells by binding to angiotensin-converting enzyme 2 (ACE2) receptor, and after virus replication and infection of the upper respiratory tract and lung tissue cells, patients begin to develop clinical symptoms and signs (12). Immunemediated inflammation such as cytokine storms and hypoxia associated with pneumonia may also cause liver injury in critically ill patients with COVID-19, which can even develop into liver failure (8).

According to reports, patients with certain viral infections (such as those caused by hepatitis $\mathrm{C}$ and human immunodeficiency virus) are more likely to develop druginduced liver injury (DILI), especially when treated with highly active anti-retroviral therapy (13). Some researchers speculate that liver injury in COVID-19 patients may also be related to drugs and may be caused by drug liver toxicity (8). Similar to SARS, antibiotics, antivirals and steroids are widely used to treat COVID-19 (14). These drugs are all potential causes of liver injury in COVID-19 patients (15). One study reported that liver injury in patients with COVID-19 may be caused by lopinavir/ritonavir, which is used as an antiviral drug for SARS-CoV-2 infection (16). Another study showed that hydroxychloroquine treatment was significantly associated with reduction/disappearance of viral load in COVID-19 patients, and azithromycin could enhance its effect (17). However, its possible impact on liver injury was not mentioned.

Overall, although there is evidence that drugs used for the treatment of COVID-19 have hepatotoxic properties (whether or not due to overdosing), the effect of antiviral treatment on liver injury is not yet clear. Therefore, this paper aimed to evaluate the effect of antiviral treatment on liver injury in critically ill patients with COVID-19. We also assessed the effect of a particular antiviral drug, arbidol, on liver injury. Confounding factors were balanced between treatment groups through propensity score matching (PSM) and inverse probability of treatment weighting (IPTW). We present the following article in accordance with the STROBE reporting checklist (available at http://dx.doi. org/10.21037/apm-20-1581).

\section{Methods}

\section{Design and study population}

This single-centered retrospective cohort study included 331 adult patients with confirmed SARS-CoV2 infection who were admitted to intensive care units (ICU) of Tongji Hospital in Wuhan, China, between January 25 and February 25, 2020. Eligible patients were required to be 22 years or older, critically ill, with complete medical records and no previous history of liver cirrhosis or hypohepatia. Critically ill patients were defined as patients admitted to ICU who required mechanical ventilation (invasive or non-invasive), or had acute respiratory distress syndrome $\left(\mathrm{PaO}_{2} / \mathrm{FiO}_{2} \leq 300 \mathrm{mmHg}\right.$; when $\mathrm{PaO}_{2}$ is not available, $\mathrm{SpO}_{2} / \mathrm{FiO}_{2} \leq 315$ indicates ARDS) or sepsis with acute organ dysfunction. The study was conducted in accordance with the Declaration of Helsinki (as revised in 2013). Ethical approval was waived by the Ethics Committee of Tongji Hospital (Wuhan, China) in view of the retrospective and observational nature of the study and all the procedures being performed were part of the routine care. And individual consent for this retrospective analysis was also waived.

The first part of the study included all eligible patients. The outcomes of patients receiving antiviral treatment were compared with those not receiving antiviral treatment. The antiviral treatment here refers to the use of oseltamivir, arbidol, lopinavir/ritonavir tablet, ganciclovir, $\alpha$-interferon and other antiviral drugs for monotherapy or combination therapy. In the second part of the study, the outcomes of patients who received arbidol treatment alone were compared with those who did not receive antiviral treatment. In this part, patients who received combination therapy or other antiviral drugs were excluded.

\section{Outcome variable}

The outcome variable was liver injury, referring to the significant abnormality in liver function indicators 
(including ALT, AST, TBIL, etc.) during the occurrence, development and treatment of COVID-19.

\section{Study covariates}

Covariates included patient demographic information (age, gender), signs and symptoms (fever, fatigue, cough, anorexia, dyspnea, expectoration, diarrhea), original comorbidities (hypertension, diabetes, CHD, COPD, cardiac insufficiency), vital signs (breathing, pulse, SBP, DBP, $\mathrm{SpO}_{2} / \mathrm{FiO}_{2}$ ), laboratory findings (ALT, AST, ALB, TBIL, LDH), organ function damage (ARDS, septic shock, DIC, AKI, myocardial damage) and treatment (anti-bacteria, gamma globulin, muscle relaxant, hormone, MV, oxygen inhalation).

\section{Statistical analyses}

Given the observational nature of the study, PSM was used to minimize the effect of potential confounders. In PSM, we used R package MatchIt (18) with full matching method, a particular type of subclassification that forms the subclasses in an optimal way (19). A fully matched sample is composed of matched sets, where each matched set contains one treated unit and one or more controls (or one control unit and one or more treated units) (20). Full matching is optimal in terms of minimizing a weighted average of the estimated distance measure between each treated subject and each control subject within each subclass. In addition, we conducted
IPTW to strictly adjust the characteristics and confounding factors that were significantly different among the patients. The stable PS inverse weights were calculated (21). In primary analysis, a survey-weighted generalized linear model was used to compare outcome variable in the IPTW cohort. The propensity score-matched analysis was applied as secondary analysis. After adjusting for differences in baseline characteristics, the effect of antiviral treatment on outcome variable was analyzed by multivariate logistic regression with adjustment for covariates, and we calculated the adjusted odds ratio (OR).

Clinical variables were expressed as frequencies and percentages (\%) for categorical variables, and median with interquartile rate (IQR) for continuous variables. We used the Pearson chi-square test for categorical data, independent two-sample $t$ test for parametric continuous data and Mann-Whitney $U$ test for non-normal continuous data. A two-sided $\mathrm{P}<0.05$ was regarded as statistically significant. No imputation was made for missing data. All statistical analyses were conducted with $\mathrm{R}$ (version 3.6.3).

\section{Results}

\section{Cobort study}

A total of 331 critically ill patients with SARS-CoV2 were included in the analysis. The baseline clinical characteristics of all patients are shown in Table 1. The median age was 64 [52-72] years, and $48.3 \%$ were male. The most common comorbidities were hypertension $(136 ; 41.1 \%)$, CHD $(66 ; 19.9 \%)$ and

Table 1 Baseline characteristics for antiviral treatment and Non-antiviral treatment groups and the whole critically ill patients with COVID-19

\begin{tabular}{|c|c|c|c|c|}
\hline & \multicolumn{4}{|c|}{ Cohort study } \\
\hline & Non-antiviral $(\mathrm{n}=58)$ & Antiviral $(n=273)$ & Total $(n=331)$ & $P$ \\
\hline Age, years, median [IQR] & 69 [57-77] & $63[51-71]$ & 64 [52-72] & 0.01 \\
\hline Gender, male, \% & $28(48.3)$ & $132(48.4)$ & $160(48.3)$ & 1 \\
\hline \multicolumn{5}{|l|}{ Signs and symptoms, \% } \\
\hline Fever & $50(86.2)$ & $237(86.8)$ & $287(86.7)$ & 1 \\
\hline Fatigue & $28(48.3)$ & $136(49.8)$ & $164(49.5)$ & 0.95 \\
\hline Cough & $39(67.2)$ & $185(67.8)$ & $224(67.7)$ & 1 \\
\hline Anorexia & $14(24.1)$ & $72(26.4)$ & $86(26.0)$ & 0.85 \\
\hline Dyspnea & $42(72.4)$ & $157(57.5)$ & $53(16.0)$ & 0.05 \\
\hline Expectoration & $20(34.5)$ & $112(41.0)$ & $199(60.1)$ & 0.44 \\
\hline Diarrhea & $16(27.6)$ & $75(27.5)$ & $132(39.9)$ & 1 \\
\hline
\end{tabular}

Table 1 (continued) 
Table 1 (continued)

\begin{tabular}{|c|c|c|c|c|}
\hline & \multicolumn{4}{|c|}{ Cohort study } \\
\hline \multicolumn{5}{|l|}{ Original comorbidities, \% } \\
\hline Hypertension & $23(39.7)$ & $113(41.4)$ & $136(41.1)$ & 0.92 \\
\hline Diabetes & $14(24.1)$ & $52(19.0)$ & $60(18.1)$ & 0.48 \\
\hline COPD & $3(5.2)$ & $13(4.8)$ & $38(11.5)$ & 1 \\
\hline Cardiac insufficiency & $6(10.3)$ & $7(2.6)$ & $16(4.8)$ & 0.02 \\
\hline \multicolumn{5}{|l|}{ Vital signs, median (IQR) } \\
\hline Breathing, rpm & 22 [20-30] & 21 [20-25] & 21 [20-25] & 0.05 \\
\hline $\mathrm{SpO}_{2} / \mathrm{FiO}_{2}$ & $166.3(96.8-287.1)$ & $290.9(222.0-337.9)$ & 284.9 (171.9-327.6) & $<0.01$ \\
\hline \multicolumn{5}{|c|}{ Laboratory findings, median (IQR) } \\
\hline ALT, $\mu / L$ & 29.5 (16.0-43.3) & $23.0(15.0-37.0)$ & $23.0(15.0-37.5)$ & 0.18 \\
\hline AST, $\mu / L$ & $39.5(25.3-65.6)$ & $30.0(20.0-45.0)$ & $30.0(21.5-46.5)$ & $<0.01$ \\
\hline ALB, g/L & $31.9(27.3-35.0)$ & $34.9(31.0-37.6)$ & $34.5(30.6-37.1)$ & $<0.01$ \\
\hline TBIL, $\mu \mathrm{mol} / \mathrm{L}$ & $10.4(7.5-21.3)$ & $9.80(7.2-13.2)$ & $9.9(7.25-13.9)$ & 0.03 \\
\hline $\mathrm{LDH}, \mathrm{U} / \mathrm{L}$ & 453.0 (320.3-735.8) & $308.0(219.0-455.0)$ & 329.0 (232.5-480.5) & $<0.01$ \\
\hline Myocardial damage & $32(55.2)$ & $72(26.4)$ & $104(31.4)$ & $<0.01$ \\
\hline Liver injury & $15(25.9)$ & $36(13.2)$ & $51(15.4)$ & 0.03 \\
\hline \multicolumn{5}{|l|}{ Treatment, \% } \\
\hline Anti-bacteria & $45(77.6)$ & $211(77.3)$ & $256(77.3)$ & 1 \\
\hline Gamma globulin & $37(63.8)$ & $109(39.9)$ & $146(44.1)$ & $<0.01$ \\
\hline Muscle relaxant & $8(13.8)$ & $29(10.6)$ & $37(11.2)$ & 0.64 \\
\hline Hormone & $43(74.1)$ & $168(61.5)$ & $211(63.7)$ & 0.10 \\
\hline MV & $36(62.1)$ & $84(30.8)$ & $120(36.3)$ & $<0.01$ \\
\hline Oxygen inhalation & $36(62.1)$ & $226(82.8)$ & 262 (79.2) & $<0.01$ \\
\hline
\end{tabular}

COPD, chronic obstructive pulmonary disease; SBP, systolic blood pressure; DBP, diastolic blood pressure; AST, aspartate aminotransferase; ALT, alanine aminotransferase; ALB, albumin; TBIL, total bilirubin; LDH, lactate dehydrogenase; ARDS, acute respiratory distress syndrome; DIC, disseminated intravascular coagulation; AKI, acute kidney injury; MV, mechanical ventilation. 
diabetes $(60 ; 18.1 \%)$. The main treatments received by patients were oxygen inhalation $(262 ; 79.2 \%)$, antibacterial treatment (256; 77.3\%), adjuvant corticosteroid treatment $(211 ; 63.7 \%)$, Gamma globulin (146; 44.1\%), mechanical ventilation (120; $36.3 \%)$ and muscle relaxant $(37 ; 11.2 \%)$. Among the 331 critically ill patients, $273(82.5 \%)$ were given antiviral therapy (including oseltamivir, arbidol, lopinavir/ritonavir, ganciclovir, interferon- $\alpha) .36(13.2 \%)$ patients with antiviral therapy developed liver injury complication.

\section{Antiviral treatment and liver injury}

As shown in Table 2, there are 36 covariates in the model.
Antiviral treatment was given to 273 (82.5\%) patients, and $58(17.5 \%)$ patients did not receive antiviral treatment. PSM and IPTW analysis were effective in controlling covariate imbalances. All covariates between the antiviral treatment and Non-antiviral treatment groups were well balanced $(\mathrm{P}>0.05)$.

Table 3 summarized the estimated effects of antiviral treatment on liver injury in critically ill patients with COVID-19. The table shows both the PSM (OR =2.77; 95\% CI: $1.03,7.48 ; \mathrm{P}=0.045)$ and the IPTW-adjusted $(\mathrm{OR}=2.33 ; 95 \% \mathrm{CI}: 1.02,5.34 ; \mathrm{P}=0.047)$ results. The two methods reached a consistent conclusion that COVID-19 critically ill patients receiving antiviral therapy had a

Table 2 Baseline characteristics for antiviral treatment and Non-antiviral treatment groups after PSM and IPTW

\begin{tabular}{|c|c|c|c|c|c|c|}
\hline & \multicolumn{3}{|c|}{ PSM } & \multicolumn{3}{|c|}{ IPTW } \\
\hline Age, years, median [IQR] & $57[46-80]$ & $63[51-71]$ & 0.87 & $57[50-78]$ & $64[52-71]$ & 0.97 \\
\hline Gender, male, \% & $28.0(48.3)$ & $132.0(48.4)$ & 0.99 & $125.6(31.7)$ & $156.5(48.2)$ & 0.11 \\
\hline \multicolumn{7}{|l|}{ Signs and symptoms, \% } \\
\hline Fatigue & $32.5(56.0)$ & $136.0(49.8)$ & 0.61 & $194.7(49.2)$ & $161.7(49.8)$ & 0.96 \\
\hline Cough & $35.3(60.9)$ & $185.0(67.8)$ & 0.55 & $233.9(59.1)$ & $220.3(67.9)$ & 0.48 \\
\hline Anorexia & $10.2(17.6)$ & $72.0(26.4)$ & 0.38 & $98.7(24.9)$ & $85.1(26.2)$ & 0.92 \\
\hline Dyspnea & $39.1(67.4)$ & $157.0(57.5)$ & 0.40 & $275.2(69.5)$ & $193.7(59.7)$ & 0.39 \\
\hline \multicolumn{7}{|l|}{ Original comorbidities, \% } \\
\hline Hypertension & $19.0(32.8)$ & $113.0(41.4)$ & 0.43 & $167.4(42.3)$ & $137.3(42.3)$ & 1 \\
\hline Diabetes & $9.9(17.0)$ & $52.0(19.0)$ & 0.83 & $64.5(16.3)$ & $65.1(20.1)$ & 0.63 \\
\hline Coronary & $3.4(5.9)$ & $29.0(10.6)$ & 0.32 & $29.0(7.3)$ & $35.8(11.0)$ & 0.44 \\
\hline COPD & $1.2(2.0)$ & $13.0(4.8)$ & 0.28 & $7.8(2.0)$ & $15.6(4.8)$ & 0.17 \\
\hline Cardiac insufficiency & $1.3(2.2)$ & $7.0(2.6)$ & 0.85 & $11.6(2.9)$ & $11.8(3.6)$ & 0.73 \\
\hline \multicolumn{7}{|l|}{ Vital signs, median (IQR) } \\
\hline Breathing, rpm & 20 [20-25] & 21 [20-25] & 0.99 & 20 [20-23] & 21 [20-25] & 0.32 \\
\hline
\end{tabular}

Table 2 (continued) 
Table 2 (continued)

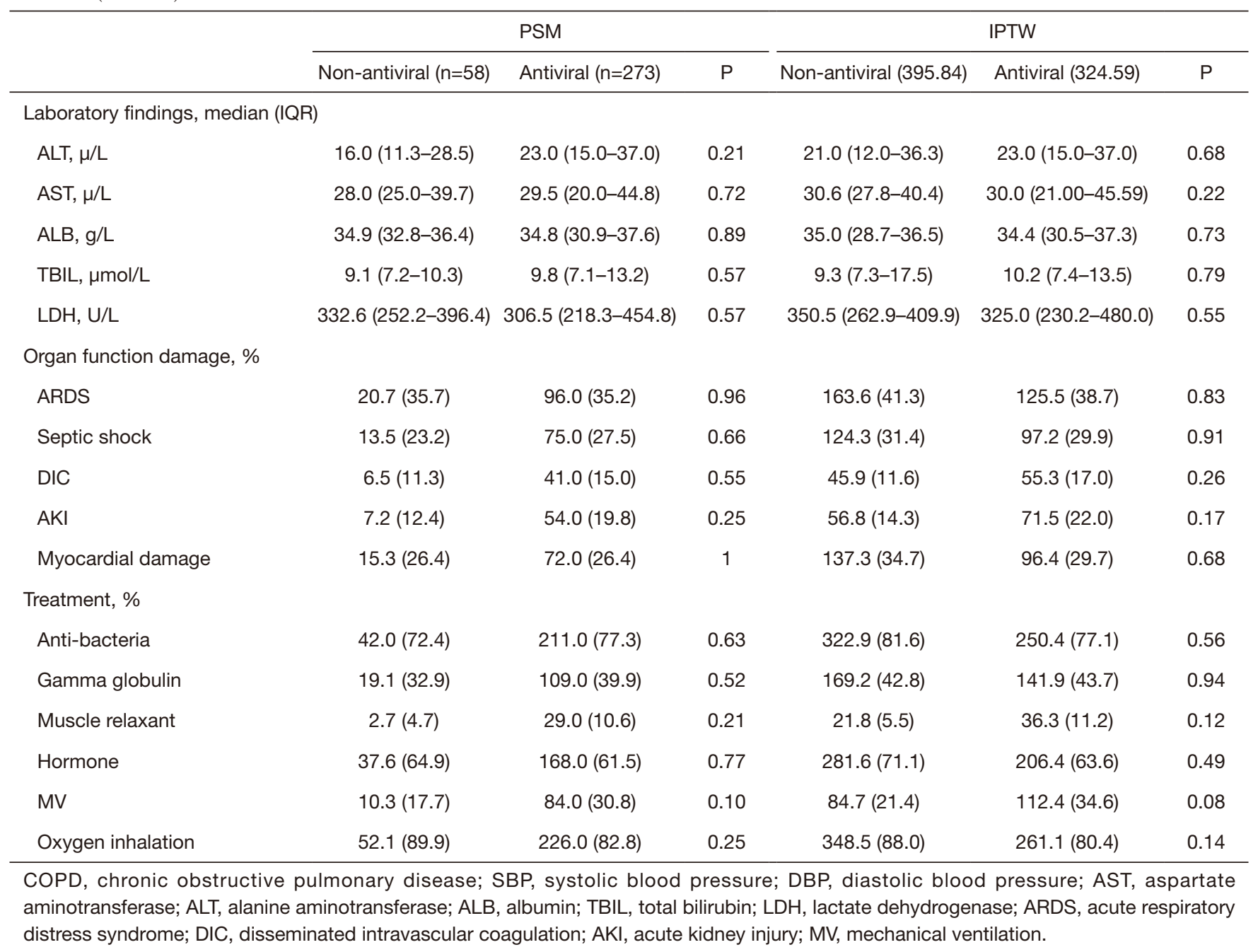

Table 3 Adjusted risk for antiviral treatment and Non-antiviral treatment groups

\begin{tabular}{llll}
\hline & OR & $95 \% \mathrm{Cl}$ & $\mathrm{P}$ \\
\hline PSM regression & 2.77 & $(1.03,7.48)$ & 0.045 \\
IPTW regression & 2.33 & $(1.02,5.34)$ & 0.047 \\
\hline
\end{tabular}

OR, odds ratio; 95\% Cl, $95 \%$ confidence interval; PSM, property score matching; IPTW, inverse probability of treatment weighting;

$\mathrm{P}$ values were determined by using the $\chi^{2}$ test.

significantly higher risk of liver injury.

\section{Arbidol treatment and liver injury}

Among the 36 covariates included in the model, 34 covariates were well balanced $(\mathrm{P}>0.05)$ between the two groups after matching, except for $\mathrm{COPD}(\mathrm{P}<0.01)$ and $\mathrm{MV}$
$(\mathrm{P}=0.04)$. After IPTW, we found that all baseline covariates between the arbidol treatment and Non-arbidol treatment groups were balanced $(\mathrm{P}>0.05$; Table 4$)$.

Since IPTW better balanced the confounding factors, the impact of arbidol treatment on critically ill patients with COVID-19 was estimated by odds ratio obtained from a regression model with IPTW. The results of 82 
Table 4 Baseline characteristics for arbidol treatment and Non- arbidol treatment groups after PSM and IPTW

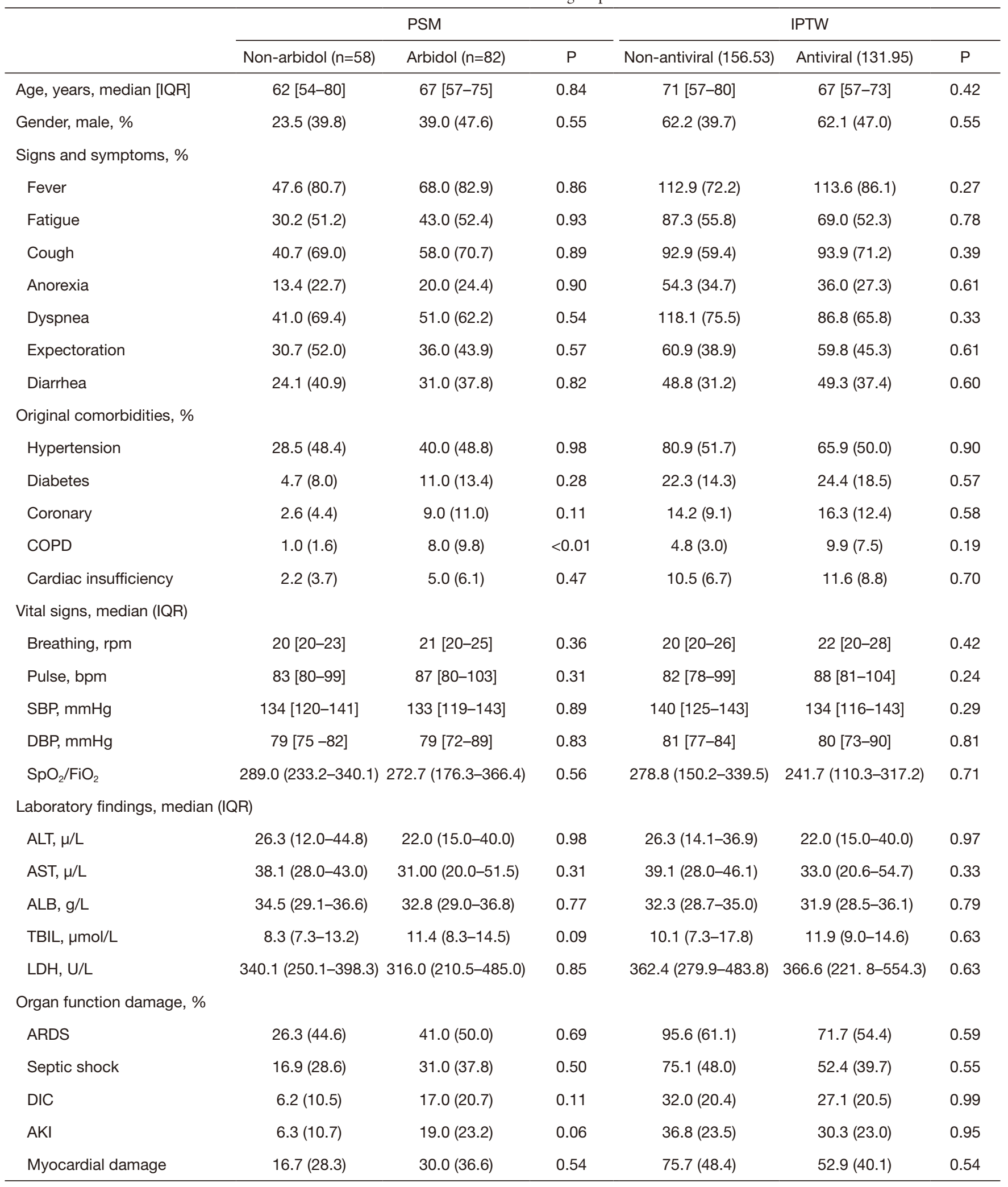

Table 4 (continued) 
Table 4 (continued)

\begin{tabular}{|c|c|c|c|c|c|c|}
\hline & \multicolumn{3}{|c|}{ PSM } & \multicolumn{3}{|c|}{ IPTW } \\
\hline \multicolumn{7}{|l|}{ Treatment, \% } \\
\hline Anti-bacteria & $42.1(71.3)$ & $69.0(84.1)$ & 0.23 & $127.0(81.1)$ & $108.3(82.1)$ & 0.91 \\
\hline Gamma globulin & $23.8(40.4)$ & $36.0(43.9)$ & 0.79 & $92.5(59.1)$ & $69.0(52.3)$ & 0.59 \\
\hline Hormone & $36.9(62.6)$ & $52.0(63.4)$ & 0.95 & $119.9(76.6)$ & $91.3(69.2)$ & 0.44 \\
\hline MV & $13.8(23.4)$ & $36.0(43.9)$ & 0.04 & $58.2(37.2)$ & $62.4(47.3)$ & 0.39 \\
\hline Oxygen inhalation & $53.6(90.9)$ & $68.0(82.9)$ & 0.17 & $125.5(80.2)$ & $102.3(77.6)$ & 0.76 \\
\hline
\end{tabular}

COPD, chronic obstructive pulmonary disease; SBP, systolic blood pressure; DBP, diastolic blood pressure; AST, aspartate aminotransferase; ALT, alanine aminotransferase; ALB, albumin; TBIL, total bilirubin; LDH, lactate dehydrogenase; ARDS, acute respiratory distress syndrome; DIC, disseminated intravascular coagulation; AKI, acute kidney injury; MV, mechanical ventilation.

patients who received antiviral arbidol alone and 58 patients without antiviral treatment showed that arbidol treatment did not have a significant effect on liver injury in COVID-19 critically ill patients $(\mathrm{OR}=2.11 ; 95 \% \mathrm{CI}: 0.79,5.67 ; \mathrm{P}=0.14)$.

\section{Discussion}

Existing studies have shown that in COVID-19, severe cases or patients with pre-existing liver diseases have a higher risk of liver injury (22). In addition, the causes of liver injury in COVID-19 may be multifactorial. It may be due to the direct effect of the virus, immune-mediated inflammation, or drug-induced toxicity. Different types of antiviral drugs, antibiotics and steroids used to treat moderate and severe diseases may induce liver injury in COVID-19 patients. Initially recommended antiviral drugs, including lopinavir/ ritonavir, chloroquine, remdesivir, tocilizumab, and umifenovir, were potentially hepatotoxic in patients with severe COVID-19, among which lopinavir/ritonavir has been shown to be associated with the progression of liver injury $(23,24)$. Systemic immune inflammation may also lead to disease progression and can contribute to liver injury (8). Elevated levels of cytokines such as IL-1, IL-6 and TNF in the plasma can lead to a systemic cytokine storm that directly causes hepatocyte damage and results in elevation of liver aminotransferases (25). However, the mechanisms of liver injury during SARS-CoV-2 infection are not yet well understood $(26,27)$.

In this article, we evaluated the effects of antiviral treatment and the use of specific antiviral drugs on liver injury in COVID-19 critically ill patients. Our current research showed that antiviral treatment did increase the risk of primary liver injury in patients. That is to say, in the treatment of coronavirus infection, drug-induced liver injury cannot be ignored. We should use antiviral treatment with caution, after fully considering the indications and complications. From a clinical point of view, in addition to actively treating the primary disease caused by coronavirus infection, attention should also be paid to monitoring the occurrence of liver injury. Liver injury in patients with mild COVID-19 is usually reversible and can heal itself without additional treatment. However, when severe liver injury occurs, we recommend the usage of hepatoprotective drugs (8).

We acknowledged the limitations of our study. First, because we used retrospective data, we applied PSM and IPTW analysis to control for the possible bias induced by unbalanced covariables between groups, but the residual confounding effects cannot be completely ruled out. Therefore, a further randomized control study is necessary to validate our results. Second, our research was restricted to COVID-19 critically ill patients, and the sample size was not very large. By expanding the sample size, it may be possible to analyze the effects of more antiviral drugs on liver injury. Third, we only analyzed the effect of receiving antiviral treatment on liver injury, without taking into account the dosage of antiviral drugs.

In conclusion, antiviral drugs are currently widely used to treat COVID-19, but our research showed that we need to consider the adverse effects of abuse of antiviral drugs. We suggest that in some cases, critically ill patients should 
be cautiously given antiviral treatment within the effective range.

\section{Acknowledgments}

We would like to thank all the hospital staff members for their efforts in collecting the information that was used in this study, and all the patients who consented to donate their data for analysis and the medical staff members who are on the frontline of caring for patients.

Funding: This work was supported by the National Key R\&D Program of China (2019YFC1711000), the National Natural Science Foundation of China (81973145), the "Double First-Class" University Project (CPU2018GY09), and the China Postdoctoral Science Foundation (2019M651805).

\section{Footnote}

Reporting Checklist: The authors have completed the STROBE reporting checklist. Available at http://dx.doi. org/10.21037/apm-20-1581

Data Sharing Statement: Available at http://dx.doi. org/10.21037/apm-20-1581

Conflicts of Interest: All authors have completed the ICMJE uniform disclosure form (available at http://dx.doi. org/10.21037/apm-20-1581). The authors have no conflicts of interest to declare.

Ethical Statement: The authors are accountable for all aspects of the work in ensuring that questions related to the accuracy or integrity of any part of the work are appropriately investigated and resolved. The study was conducted in accordance with the Declaration of Helsinki (as revised in 2013). Ethical approval was waived by the Ethics Committee of Tongji Hospital (Wuhan, China) in view of the retrospective and observational nature of the study and all the procedures being performed were part of the routine care. And individual consent for this retrospective analysis was also waived.

Open Access Statement: This is an Open Access article distributed in accordance with the Creative Commons Attribution-NonCommercial-NoDerivs 4.0 International License (CC BY-NC-ND 4.0), which permits the noncommercial replication and distribution of the article with the strict proviso that no changes or edits are made and the original work is properly cited (including links to both the formal publication through the relevant DOI and the license). See: https://creativecommons.org/licenses/by-nc-nd/4.0/.

\section{References}

1. Zhu N, Zhang D, Wang W, et al. A novel coronavirus from patients with pneumonia in China, 2019. N Engl J Med 2020;382:727-33.

2. Guan WJ, Ni ZY, Hu Y, et al. Clinical characteristics of coronavirus disease 2019 in China. N Engl J Med 2020;382:1708-20.

3. Wang FS, Zhang C. What to do next to control the 2019nCoV epidemic? Lancet 2020;395:391-3.

4. Huang C, Wang Y, Li X, et al. Clinical features of patients infected with 2019 novel coronavirus in Wuhan, China. Lancet 2020;395:497-506.

5. Raoult D, Zumla A, Locatelli F, et al. Coronavirus infections: Epidemiological, clinical and immunological features and hypotheses. Cell Stress 2020;4:66.

6. Xu L, Liu J, Lu M, et al. Liver injury during highly pathogenic human coronavirus infections. Liver Int 2020;40:998-1004.

7. Zhou F, Yu T, Du R, et al. Clinical course and risk factors for mortality of adult inpatients with COVID-19 in Wuhan, China: a retrospective cohort study. Lancet 2020;395:1054-62.

8. Zhang C, Shi L, Wang FS. Liver injury in COVID-19: management and challenges. Lancet Gastroenterol Hepatol 2020;5:428-30.

9. Chen $\mathrm{G}, \mathrm{Wu} \mathrm{D}$, Guo $\mathrm{W}$, et al. Clinical and immunologic features in severe and moderate forms of Coronavirus Disease. J Clin Invest 2020;130:2620-9.

10. Zhang B, Zhou X, Qiu Y, et al. Clinical characteristics of 82 death cases with COVID-19. PLoS One 2020;15:e235458.

11. Yeo C, Kaushal S, Yeo D. Enteric involvement of coronaviruses: is faecal-oral transmission of SARS-CoV-2 possible? Lancet Gastroenterol Hepatol 2020;5:335-7.

12. Wang D, Hu B, Hu C, et al. Clinical characteristics of 138 hospitalized patients with 2019 novel coronavirus-infected pneumonia in Wuhan, China. JAMA 2020;323:1061-9.

13. Naidoo K, Hassan-Moosa R, Mlotshwa P, et al. High Rates of Drug-induced Liver Injury in People Living With HIV Coinfected With Tuberculosis (TB) Irrespective of Antiretroviral Therapy Timing During Antituberculosis Treatment: Results From the Starting Antiretroviral 
Therapy at Three Points in TB Trial. Clin Infect Dis 2020;70:2675-82.

14. Xu Z, Shi L, Wang Y, et al. Pathological findings of COVID-19 associated with acute respiratory distress syndrome. Lancet Respir Med 2020;8:420-2.

15. Jin YH, Cai L, Cheng ZS, et al. A rapid advice guideline for the diagnosis and treatment of 2019 novel coronavirus (2019-nCoV) infected pneumonia (standard version). Mil Med Res 2020;7:4.

16. Yang X, Yu Y, Xu J, et al. Clinical course and outcomes of critically ill patients with SARS-CoV-2 pneumonia in Wuhan, China: a single-centered, retrospective, observational study. Lancet Respir Med 2020;8:475-81.

17. Gautret P, Lagier JC, Parola P, et al. Hydroxychloroquine and azithromycin as a treatment of COVID-19: results of an open-label non-randomized clinical trial. Int J Antimicrob Agents 2020;56:105949.

18. Ho DE, Imai K, King G, et al. MatchIt: nonparametric preprocessing for parametric causal inference. J Stat Softw 2011;42:1-28.

19. Rosenbaum PR, Rubin DB. The central role of the propensity score in observational studies for causal effects. Biometrika 1983;70:41-55.

Cite this article as: Ruan X, Lu X, Wang K, Zhang B, Wang J, Li Y, Wang J, Xu Z, Yan F. Liver injury after antiviral treatment of critically ill patients with COVID-19: a single-centered retrospective cohort study. Ann Palliat Med 2021;10(3):2429-2438. doi: 10.21037/apm-20-1581
20. Hansen BB. Full matching in an observational study of coaching for the SAT. J Am Stat Assoc 2004;99:609-18.

21. Austin PC, Stuart EA. Moving towards best practice when using inverse probability of treatment weighting (IPTW) using the propensity score to estimate causal treatment effects in observational studies. Stat Med 2015;34:3661-79.

22. Ali N, Hossain K. Liver injury in severe COVID-19 infection: current insights and challenges. Expert Rev Gastroenterol Hepatol 2020;14:879-84.

23. Cai Q, Huang D, Yu H, et al. COVID-19: Abnormal liver function tests. J Hepatol 2020;73:566-74.

24. Cai Q, Huang D, Ou P, et al. COVID-19 in a designated infectious diseases hospital outside Hubei Province, China. Allergy 2020;75:1742-52.

25. Li Y, Xiao SY. Hepatic involvement in COVID-19 patients: pathology, pathogenesis and clinical implications. J Med Virol 2020;92:1491-4.

26. Medetalibeyoglu A, Catma Y, Senkal N, et al. The effect of liver test abnormalities on the prognosis of COVID-19. Ann Hepatol 2020;19:614-21.

27. Ali N. Relationship Between COVID-19 Infection and Liver Injury: A Review of Recent Data. Front Med (Lausanne) 2020;7:458. 